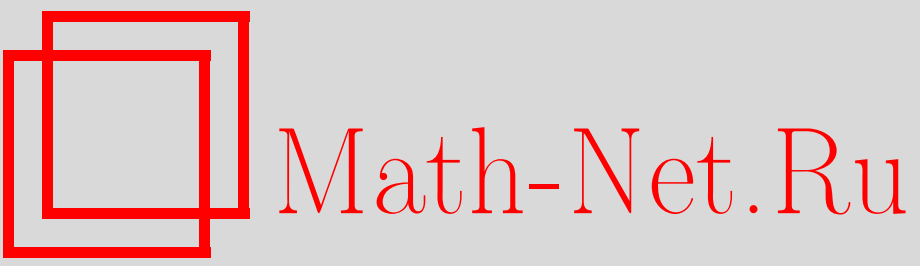

Ю. Н. Беляев, Матричный метод расчёта перерассеяния волн в периодической структуре, Вестн. Сам. гос. техн. ун-та. Сер. Физ.-мат. науки, 2011, выпуск 2(), 142-148

DOI: https://doi.org/10.14498/vsgtu854

Использование Общероссийского математического портала Math-Net.Ru подразумевает, что вы прочитали и согласны с пользовательским соглашением

http: //www. mathnet.ru/rus/agreement

Параметры загрузки:

IP : 35.174 .16 .151

26 апреля 2023 г., 13:20:26 
УДК $512.64+534.2+535.3$

\section{МАТРИЧНЫЙ МЕТОД РАСЧЁТА ПЕРЕРАССЕЯНИЯ ВОЛН В ПЕРИОДИЧЕСКОЙ СТРУКТУРЕ}

\section{Ю. Н. Беляев}

Сыктывкарский государственный университет, 167001, Сыктывкар, Октябрьский пр., 55.

E-mail: ybelyayev@mail.ru

Развит метод рекуррентного расчёта характеристической матриць слоистой структуры. Получены аналитические решения для матрии второгочетвёртого порядков, описывающих распространение волн в периодических структурах.

Ключевые слова: матрица, периодическая структура, волна, рекуррентные формуль, полиномы.

Введение. Один из матричных подходов к теории распространения волн в слоистых средах - метод характеристической матрицы был предложен в середине XX века Ф. Абеле [1] для расчёта оптических и В. Томсоном [2] и Н. Хаскелом [3] - упругих свойств слоистых сред с плоскопараллельными границами. Позднее этот метод был развит применительно к рентгеновской дифракции в слоистых кристаллах, в частности, в совершенном кристалле с нарушенным поверхностным слоем [4].

Согласно методу характеристической матрицы $i$-тый слой рассматриваемой структуры характеризуется матрицей $M_{i}$, которая связывает тангенциальные компоненты векторов волнового поля на противоположных границах слоя. Система из $N$ слоёв описывается матрицей $M=\prod_{i=1}^{N} M_{i}$, получающейся при перемножении в определённом порядке характеристических матриц всех слоёв системы. Данный метод позволяет при рассмотрении интерференционных решений обойти сложную процедуру удовлетворения всех граничных условий на межслойных границах структуры и облегчает учёт многократного перерассеяния волн между слоями. Метод характеристической матрицы позволяет с единых позиций рассматривать рассеяние акустических, электромагнитных и упругих волн в слоистых средах.

Как известно, распространение плоской световой волны в диэлектрической (или металлической) плёнке можно описать с помощью характеристической матрицы второго порядка; преобразование компонент вектора смещения и напряжения в упругом однородном слое осуществляется характеристическими матрицами 4-6 порядков, а $n$-лучевая дифракция излучения рентгеновского диапазона частот в совершенном кристалле может быть описана с помощью матрицы $n$-ного порядка. Периодическая структура, сформированная из $k$ одинаковых слоёв, описываются $k$-той степенью матрицы (соответствующего порядка) одного слоя.

В данной работе развивается метод рекуррентного расчёта степеней матрицы применительно к одномерно периодическим структурам.

Юрий Николаевич Беляев (к.ф.-м.н., доц.), декан, физико-технический факультет. 
1. Матрицы кратных линейных операций. По теореме Гамильтона-Кэли каждая матрица $M n$-ного порядка удовлетворяет своему характеристическому уравнению

$$
(-1)^{n} M^{n}+(-1)^{n-1} \sigma_{1} M^{n-1}+\cdots-\sigma_{n-1} M+\sigma_{n} I=0,
$$

где $I \equiv M^{0}$ - единичная матрица, а $\sigma_{i}$-сумма всех $C_{n}^{i}$ главных миноров $i$-того $(i=1,2, \ldots, n)$ порядка определителя матрицы $M$ (в частности, $\sigma_{1}=$ $=\operatorname{tr} M-$ след, а $\sigma_{n}=\operatorname{det} M-$ определитель матрицы $\left.M\right)$. Из формулы (1) нетрудно заметить, что целые степени квадратной матрицы $n$-ного порядка могут быть выражены через первые $n$ степеней: $M^{0} \equiv I, M, \ldots, M^{n-1}$. Справедлива

Теорема. Пусть $M-$ квадратная матрица п-ного порядка. Тогда

$$
\begin{gathered}
M^{k}=M^{n-1}\left(p_{1} B_{k-1}+\cdots+p_{n} B_{k-n}\right)+M^{n-2}\left(p_{2} B_{k-1}+\cdots+p_{n} B_{k+1-n}\right)+ \\
+\cdots+M\left(p_{n-1} B_{k-1}+p_{n} B_{k-2}\right)+I p_{n} B_{k-1}, \quad k=0,1,2, \ldots
\end{gathered}
$$

где $p_{i}=(-1)^{i-1} \sigma_{i}, i=1,2, \ldots, n$, а многочлены $B_{k}$ определяются рекуррентными формулами

$$
B_{k}=p_{1} B_{k-1}+\cdots+p_{n} B_{k-n} ; \quad B_{0}=\cdots=B_{n-3}=B_{n-2}=0, \quad B_{n-1}=1 .
$$

Непосредственной проверкой легко убедиться в справедливости соотношения (2) при $k=0,1, \ldots, n-1$. Для других $k$ равенство (2) доказывается (см. [5, стр. 63]) по индукции.

2. Метод рекуррентного расчёта степеней матрицы. Вычисление $M^{k}$ удобно проводить по формуле (2), используя рекуррентную процедуру нахождения многочленов $B_{k}$, согласно следующей последовательности действий [6]:

1) перемножением матрицы $M$ самой на себя $n-1$ раз вычисляются степени матрицы $M$ со второй по $n$-ную включительно и параллельно находится след $s_{i}$ для каждой из матриц $M^{i}(i=1,2, \ldots, n)$;

2) последовательное вычисление коэффициентов $p_{i}$ выполняется по формуле Ньютона

$$
i p_{i}=s_{i}-p_{1} s_{i-1}-\cdots-p_{i-1} s_{1}, \quad i=1,2, \ldots, n ;
$$

3) значения многочленов $B_{j}(j=1,2, \ldots, n)$ определяются по рекуррентным формулам (3).

Несложно оценить трудоёмкость данной процедуры по сравнению с обычным перемножением матриц. Число операций сложения и умножения, необходимое для возведения матрицы порядка $n$ в $k$-тую степень $(k>n)$ по предлагаемому в данной работе методу не превышает $N_{1}=n[(n-1)(2 n+k-3)+$ $+2 n+1]$. Если же вычислять $M^{k}$ как $k-1$ произведение матриц $M$, то это потребует $N_{2}=n^{2}(2 n-1)(k-1)$ операций сложения и умножения.

Уже для структур с 25 слоями, описываемых характеристическими матрицами 4-6 порядков, $N_{2} / N_{1}>3$, т. е. метод рекуррентного расчёта характеристической матрицы не менее чем в три раза эффективнее (по числу операций и, следовательно, по точности счёта), чем обычное перемножение. А для 
структуры, состоящей из 250 слоёв, $N_{2} / N_{1}>8,9,10$ соответственно для матриц 4,5 и 6 порядков.

Для характеристических уравнений матриц 2-4 порядков имеются аналитические решения. Рассмотрим ряд случаев, в которых элементы характеристической матрицы периодической структуры допускают аналитическое представление через элементы матрицы одного периода.

3. Матрицы второго порядка. При $n=2$ из формул (2) и (3) находим

$$
M^{k}=B_{k} M-\sigma_{2} B_{k-1} I,
$$

где

$$
B_{k}=\sigma_{1} B_{k-1}-\sigma_{2} B_{k-2}, \quad B_{0}=0, \quad B_{1}=1 .
$$

Коэффициенты $\sigma_{i}$ характеристического уравнения связаны с собственными числами $\lambda_{1}, \lambda_{2}$ матрицы $M$ :

$$
\sigma_{1}=\lambda_{1}+\lambda_{2}, \quad \sigma_{2}=\lambda_{1} \lambda_{2} \Longleftrightarrow \lambda_{1,2}=\frac{\sigma_{1}}{2} \pm \sqrt{\left(\frac{\sigma_{1}}{2}\right)^{2}-\sigma_{2}} .
$$

Решением уравнений (4) являются

$$
B_{k}=\frac{\lambda_{2}^{k}-\lambda_{1}^{k}}{\lambda_{2}-\lambda_{1}}=\frac{\left(\frac{\sigma_{1}}{2}+\sqrt{\left(\frac{\sigma_{1}}{2}\right)^{2}-\sigma_{2}}\right)^{k}-\left(\frac{\sigma_{1}}{2}-\sqrt{\left(\frac{\sigma_{1}}{2}\right)^{2}-\sigma_{2}}\right)^{k}}{2 \sqrt{\left(\frac{\sigma_{1}}{2}\right)^{2}-\sigma_{2}}}
$$

ЗАмечание. Численный расчёт собственных многочленов $B_{k}$ более точно и менее трудоёмко (по числу операций сложения и умножения) выполняется по рекуррентным соотношениям (4), чем по соответствующей формуле $(5)$, явно выражающей многочлены $B_{k}$ через собственные значения матрицы. Аналогичные утверждения справедливы и для матриц больших порядков.

Если матрица $M$ не вырожденная $\left(\sigma_{2} \neq 0\right)$, её степени допускают следующее представление:

$$
M^{k}=\left\|m_{i j}\right\|^{k}=\sigma_{2}^{\frac{k}{2}}\left\|\begin{array}{cc}
\frac{m_{11}}{\sqrt{\sigma_{2}}} U_{k-1}(b)-U_{k-2}(b) & \frac{m_{12}}{\sqrt{\sigma_{2}}} U_{k-1}(b) \\
\frac{m_{21}}{\sqrt{\sigma_{2}}} U_{k-1}(b) & \frac{m_{22}}{\sqrt{\sigma_{2}}} U_{k-1}(b)-U_{k-2}(b)
\end{array}\right\|,
$$

где $b=\sigma_{1} /\left(2 \sqrt{\sigma_{2}}\right)$, а $U_{k}$ - полиномы, удовлетворяющие рекуррентным формулам:

$$
U_{0}=1, \quad U_{1}=2 b, \quad U_{k}(b)=2 b U_{k-1}(b)-U_{k-2}(b) .
$$

Если $-1 \leqslant b \leqslant 1$, функции, определяемые соотношениями (7), являются ортогональными полиномами Чебышёва второго рода:

$$
U_{k}(b)=\frac{\sin [(k+1) \varphi]}{\sin \varphi}, \quad b=\cos \varphi
$$


ПримеР 1. При нормальном падении видимого света длиной волны $\lambda_{0}$ на многослойную оптическую систему, образованную чередованием однородных плёнок, система характеризуется матрицей (6), в которой

$$
\sigma_{2}=1, \quad b=\cos \beta_{2} \cos \beta_{3}, \quad \beta_{2}=\left(2 \pi / \lambda_{0}\right) n_{2} h_{2}, \quad \beta_{3}=\left(2 \pi / \lambda_{0}\right) n_{3} h_{3},
$$

где $n_{2}, n_{3}$ - показатели преломления, а $h_{2}, h_{3}$ - толщины слоёв.

ПримеР 2. Для симметричного брэгговского отражения рентгеновской монохроматической волны от тонкой кристаллической плёнки характеристическая матрица также будет унимодулярной $\left(\sigma_{2}=1\right)$, а аргумент полиномов Чебышёва выражается через отстройку $\Delta \theta$ угла падения от точного угла Брэгга $\theta_{0}$ и расстояние $a$ между атомными плоскостями, находящимися в отражающем положении: $b=\cos \left(\left(2 \pi / \lambda_{0}\right) a \cos \theta_{0} \Delta \theta\right)$.

4. Матрицы третьего порядка. Для этого случая из формул (2)-(3) получаем

$$
M^{k}=M^{2} B_{k}+M\left(-\sigma_{2} B_{k-1}+\sigma_{3} B_{k-2}\right)+I \sigma_{3} B_{k-1},
$$

где

$$
B_{k}=\sigma_{1} B_{k-1}-\sigma_{2} B_{k-2}+\sigma_{3} B_{k-3}, \quad B_{0}=B_{1}=0, \quad B_{2}=1 .
$$

Решением уравнений (9) являются многочлены

$$
B_{k} \equiv \frac{\lambda_{1}^{k} \lambda_{3}-\lambda_{1}^{k} \lambda_{2}+\lambda_{2}^{k} \lambda_{1}-\lambda_{2}^{k} \lambda_{3}+\lambda_{3}^{k} \lambda_{2}-\lambda_{3}^{k} \lambda_{1}}{\lambda_{1}^{2} \lambda_{3}-\lambda_{1}^{2} \lambda_{2}+\lambda_{2}^{2} \lambda_{1}-\lambda_{2}^{2} \lambda_{3}+\lambda_{3}^{2} \lambda_{2}-\lambda_{3}^{2} \lambda_{1}}
$$

где $\lambda_{1}, \lambda_{2}, \lambda_{3}$ - корни характеристического уравнения, соответствующего матрице третьего порядка.

Аналитические выражения для степеней матриц третьего порядка с помощью ортогональных полиномов возможны при специальном виде этих матриц. В частности, не представляет труда получение соответствующих выражений для вырожденных матриц. Другая возможность, которая может реализоваться не только для матриц третьего, но и более высоких порядков, возникает, когда вторая степень матрицы (любого порядка) является линейной комбинацией самой матрицы и единичной матрицы (см. [5, стр. 46]).

5. Матрицы четвёртого порядка. Собственные многочлены $B_{k}$ матрицы $M$ четвёртого порядка в общем случае удовлетворяют рекуррентным формулам

$$
B_{0}=B_{1}=B_{2}=0, \quad B_{3}=1, \quad B_{k}=\sigma_{1} B_{k-1}-\sigma_{2} B_{k-2}+\sigma_{3} B_{k-3}-\sigma_{4} B_{k-4},
$$

а степени матрицы -

$$
\begin{aligned}
M^{k}=M^{3}\left(\sigma_{1} B_{k-1}-\right. & \left.\sigma_{2} B_{k-2}+\sigma_{3} B_{k-3}-\sigma_{4} B_{k-4}\right)- \\
-M^{2}\left(\sigma_{2} B_{k-1}-\right. & \left.\sigma_{3} B_{k-2}+\sigma_{4} B_{k-3}\right)+ \\
& +M\left(\sigma_{3} B_{k-1}-\sigma_{4} B_{k-2}\right)-I \sigma_{4} B_{k-1} .
\end{aligned}
$$

Рассмотрим частный случай. Пусть

$$
\sigma_{1}=\sigma_{3}=0, \quad \sigma_{2} \neq 0, \quad \sigma_{4} \neq 0 .
$$


Тогда все многочлены $B_{k}$ с чётными индексами равны нулю, а многочлены с нечётными индексами определяются равенствами

$$
B_{1}=0, \quad B_{3}=1, \quad B_{2 k+1}=-\sigma_{2} B_{2 k-1}-\sigma_{4} B_{2 k-3} .
$$

Для степеней матрицы $M$ находим два эквивалентных представления:

$$
\begin{gathered}
M^{k}= \begin{cases}M^{2} B_{k+1}-I \sigma_{4} B_{k-1}, & k=0,2,4, \ldots, \\
M^{3} B_{k}-M \sigma_{4} B_{k-2}, & k=1,3,5, \ldots ;\end{cases} \\
M^{k}=\left\{\begin{array}{ll}
M^{2} \sigma_{4}^{\frac{j-1}{2}} U_{j-1}-I \sigma_{4}^{\frac{j}{2}} U_{j-2}, \quad k=2 j, \\
M^{3} \sigma_{4}^{\frac{j-1}{2}} U_{j-1}-M \sigma_{4}^{\frac{j}{2}} U_{j-2}, & k=2 j+1,
\end{array} \quad j=0,1,2, \ldots .\right.
\end{gathered}
$$

Здесь $U_{j}=U_{j}(b)$ - полиномы, определяемые рекуррентными формулами (7), с аргументом $b=-\sigma_{2} /\left(2 \sqrt{\sigma_{4}}\right)$.

ПРИМЕР 3 (ХАРАКТЕРИСТИЧЕСКАЯ МАТРИЦА НЕОДНОРОДНОГО ПО ТОЛЩИНЕ слоя). Слой материала, упругие свойства которого меняются по толщине, можно аппроксимировать пачкой слоёв, каждый из которых является однородным. Соответственно характеристическая матрица такой системы будет равна произведению характеристических матриц отдельных слоёв. Характеристическая матрица однородного упругого слоя в подходе Томсона-Хаскела может быть представлена в виде [7]:

$$
M=\exp (\kappa h \chi), \quad \chi=\left\|\begin{array}{cccc}
0 & -1 & d & 0 \\
b & 0 & 0 & c \\
p \eta^{2}+a & 0 & 0 & -b \\
0 & p \eta^{2} & 1 & 0
\end{array}\right\|
$$

где

$$
a=\frac{4 \mu(\lambda+\mu)}{\lambda+2 \mu}, \quad b=\frac{\lambda}{\lambda+2 \mu}, \quad c=\frac{1}{\lambda+2 \mu}, \quad d=\frac{1}{\mu}
$$

$h$ - толщина слоя, $\rho$ - плотность вещества, $\lambda$ и $\mu$-коэффициенты Ламэ, $\kappa$ и $\eta$ - параметры интегрирования.

Матрица $\chi$ удовлетворяет условиям (12). А именно,

$$
\sigma_{2}=-\frac{3-4 \nu}{1-\nu} x-2, \quad \sigma_{4}=\frac{2(1+\nu)^{2}(1-2 \nu)}{\nu} x^{2}+\frac{4\left(\nu^{2}-7 \nu+3\right)(1+\nu)}{(1-\nu)^{2}} x+1
$$

где $x=\rho \eta^{2} / E, \nu$ - коэффициент Пуассона, $E$ - модуль Юнга.

Используя полученный ранее результат (13), выражение для характеристической матрицы однородного слоя можно представить в следующем виде:

$$
M=\alpha I+\beta \chi+\gamma \chi^{2}+\delta \chi^{3},
$$


где

$$
\begin{aligned}
& \alpha=1-\sigma_{4}\left[B_{3} \frac{(\kappa h)^{4}}{4 !}+B_{5} \frac{(\kappa h)^{6}}{6 !}+\ldots\right], \\
& \gamma=\frac{(\kappa h)^{2}}{2 !}+B_{5} \frac{(\kappa h)^{4}}{4 !}+B_{7} \frac{(\kappa h)^{6}}{6 !}+\ldots, \\
& \beta=\kappa h-\sigma_{4}\left[B_{3} \frac{(\kappa h)^{5}}{5 !}+B_{5} \frac{(\kappa h)^{7}}{7 !}+\ldots\right], \\
& \delta=\frac{(\kappa h)^{3}}{3 !}+B_{5} \frac{(\kappa h)^{5}}{5 !}+B_{7} \frac{(\kappa h)^{7}}{7 !}+\ldots
\end{aligned}
$$

Соответственно матрица неоднородного слоя есть

$$
\prod_{i} M_{i}=\prod_{i}\left(\alpha_{i} I+\beta_{i} \chi_{i}+\gamma_{i} \chi_{i}^{2}+\delta_{i} \chi_{i}^{3}\right) .
$$

Из формул (16) и (15) несложно получить выражение для характеристической матрицы неоднородной структуры с любой заданной точностью по величинам толщин $h_{i}$ отдельных слоёв. В качестве иллюстрации построим характеристическую матрицу идеальной тонкой кристаллической плёнки. Для этого воспользуемся моделью, представляющей кристалл как дискретно периодическую структуру, в которой атомная плоскость и межатомный слой представляются двумя однородными упругими средами, имеющими различные постоянные упругости. Будем отмечать все величины, относящиеся к этим средам, индексами 1 и 2 . В результате для элементов $C_{i j}$ характеристической матрицы всей структуры, содержащей $N$ периодов, с точностью до величин первого порядка малости по толщинам $h_{1}$ и $h_{2}$ получим следующие выражения:

$$
\begin{gathered}
C_{11}=C_{22}=C_{33}=C_{44}=1, \quad C_{41}=C_{32}=C_{23}=C_{14}=0 \\
C_{13}=N \kappa\left(d_{1} h_{1}+d_{2} h_{2}\right), \quad C_{43}=-C_{12}=N \kappa\left(h_{1}+h_{2}\right) \\
C_{24}=N \kappa\left(c_{1} h_{1}+c_{2} h_{2}\right), \quad C_{42}=N \kappa \eta^{2}\left(\rho_{1} h_{1}+\rho_{2} h_{2}\right) \\
C_{31}=N \kappa \eta^{2}\left(\rho_{1} h_{1}+\rho_{2} h_{2}\right)+N \kappa\left(a_{1} h_{1}+a_{2} h_{2}\right) \\
C_{21}=-C_{34}=N \kappa\left(b_{1} h_{1}+b_{2} h_{2}\right) .
\end{gathered}
$$

Заключение. Отметим существенное преимущество развиваемого в данной работе метода рекуррентного расчёта степеней матрицы по сравнению с алгоритмом вычисления степеней матрицы по формуле Лагранжа-Сильвестра [8]. Первый метод в отличие от второго не требует вычисления собственных значений матрицы $M$ для нахождения $M^{k}$, что особенно важно для матриц, порядок которых выше 4.

Работа выполнена при поддержке ФЦП «Научные и научно-педагогические кадры инновационной России» на 2009-2013 годы (гос. контракт № 02.740.11.0618).

\section{БИБЛИОГРАФИЧЕСКИЙ СПИСОК}

1. Abelès $F$. Recherehes sur la propagation des ondes électromagnétiques sinusoïdals dans les milieux stratifiés. Application aux conches minces // Ann. Physique, 1950. Vol. 5. Pp. 596640. 
2. Thomson W. T. Transmission of elastic wave through a stratified solid material // J. Appl. Phys., 1950. Vol.21, no. 1. Pp. 89-93.

3. Haskell N. A. The dispersion of surface waves on multi-layered media// Bul. Seismol. Soc. Amer., 1953. Vol. 43, no. 1. Pp. 17-34.

4. Belyaev Yu. N., Kolpakov A.V. On the theory of X-ray diffraction in a perfect crystal with distorted surface layer // Physica Status Solidi (a), 1983. Vol.76, no. 2. Pp. 641-646.

5. Беляев Ю. Н. Алгебра тензоров. Сыктывкар: СыктГУ, 2009. 180 с. [Belyayev Yu. N. The Algebra of tensors. Syktyvkar: SyktGU, 2009. 180 pp.]

6. Беллев Ю. Н. Характеристическая матрица слоисто-периодической структуры // Вестн. Сыктывкар. ун-та. Сер. 1, 2010. №11. С. 86-91. [Belyayev Yu. N. Characteristic matrix of laered-periodic structure // Vestn. Syktyvkar. un-ta. Ser. 1., 2010. no. 11. Pp. 86-91].

7. Молотков Л. А. Матричный метод в теории распространения волн в слоистых упругих и жидких средах. Ленинград: Наука, 1984. 201 с. [Molotkov L. A. The matrix method in the theory of wave propagation in layered elastic and liquid media. Leningrad: Nauka, 1984. 201 pp.]

8. Гантмахер Ф.Р. Теория матриц. М.: Наука, 1988. 549 с. [Gantmakher F. R. Theory of matrices. Moscow: Nauka, 1988. 549 pp.]

Поступила в редакцию 20/XII/2010;

в окончательном варианте - 9/III/2011.

MSC: 78A45; 15A16, 74J20

\section{MATRIX METHOD OF CALCULATING THE RESCATTERING WAVE IN A PERIODIC STRUCTURE}

\section{Yu. N. Belyayev}

Syktyvrar State University,

55, Oktyabrskiy pr., Syktyvkar, 167001, Russia.

E-mail: ybelyayev@mail.ru

The method of recursive calculation of the characteristic matrix of the layered structure is developed. Analytical solutions for the matrix of second, third and fourth order describing the propagation of waves in periodic structures are found.

Key words: matrix, periodic structure, wave, recurrence fomula, polynomials.

Original article submitted 20/XII/2010; revision submitted $9 / \mathrm{III} / 2011$.

Yuriy N. Belyayev (Ph. D. (Phys. \& Math.)), Dean, Faculty of Physics and Technology. 